RESEARCH ARTICLE

\title{
Porphyrin-sensitized photo-oxidation of hematoxylin in oxygenated solutions
}

\author{
Ajith Herath, Namal Priyantha, Gamini Rajapakse, Veranja Karunaratne and Anura \\ Wickramasinghe* \\ Department of Chemistry, Faculty of Science, University of Peradeniya, Peradeniya.
}

Revised:18 January 2007 ; Accepted:21 May 2007

\begin{abstract}
This paper describes the oxidation of hematoxylin (HT) in oxygenated aqueous solutions at $\mathrm{pH} 4.0$, upon irradiation with visible light, in the presence of protoporphyrin dimethylester (PPDME) as a photosensitizer. The redox potential for the lowest excited state of PPDME, estimated from its ground state oxidation potential $[0.86 \mathrm{~V}$ with respect to normal hydrogen electrode (NHE)] and its low energy absorption edge $(2.5 \mathrm{eV})$, was found to be $-1.63 \mathrm{~V}$ (with respect to NHE). These values clearly indicate that the excited PPDME molecules could transfer their energy to the ground state oxygen in solution, producing the superoxide radical anion $\left(\mathrm{O}_{2}{ }^{--}\right)$or singlet oxygen. Additional experiments carried out in the presence of sodium azide and/or benzoquinone, confirm that the oxidation of HT mainly proceeds through a superoxide radical based mechanism. Under the conditions described above, HT was found to be completely oxidised upon 24 hours of visible light irradiation.
\end{abstract}

Keywords: Hematoxylin, irradiation, photo-oxidation, photosensitizer, protoporphyrin dimethylester, superoxide anion, visible light.

\section{INTRODUCTION}

Non-biodegradable organic pollutants present in the environment have been a growing threat to human and aquatic life ${ }^{1}$. Traditional methods of pollution abatement, such as adsorption, coagulation and biological treatment, were shown to be less effective in removing such organic pollutants in waste-waters. Considerable attention has, therefore, been focused on the UV photolysis of organic substances in the presence of oxidants such as $\mathrm{H}_{2} \mathrm{O}_{2}, \mathrm{O}_{3}{ }^{2-4}$ and semiconductor photocatalysts ${ }^{5-7}$. Photosensitized oxidative degradation of organic compounds in the aquatic systems, has been proven to be a highly promising method for the conversion of such organic pollutants into less harmful substances ${ }^{8-10}$. Photosensitizers such as porphyrin analogues, phthalocyanins and dyes such as methylene blue, rose bengal etc., in combination with visible light, in oxygenated solutions, generate highly potent oxidizing species such as singlet oxygen $\left({ }^{1} \Delta_{\mathrm{g}}\right)$ and/or the superoxide anion $\left(\mathrm{O}_{2}{ }^{-}\right)$. Such photosensitizers are, therefore, of great importance for the oxidative degradation of organic pollutants.

A photosensitizer (PS), by absorbing the correct energy from the visible spectrum, gets excited to its lowest excited singlet state $\left({ }^{1} \mathrm{PS}^{*}\right)$. The latter then follows the intersystem crossing (ISC) to the triplet excited state $\left({ }^{3} \mathrm{PS}^{*}\right)$ of the sensitizer. If the energy of this triplet state is around $94 \mathrm{~kJ} \mathrm{~mol}^{-1}$, it can then be quenched by the ground state triplet oxygen $\mathrm{O}_{2}\left({ }^{3} \sum_{\mathrm{g}}^{-}\right)$, forming the lowest excited singlet state of oxygen $\left({ }^{1} \Delta_{\mathrm{g}}\right)$ and the second excited singlet state of oxygen $\left({ }^{1} \sum_{\mathrm{g}}^{+}\right)$, through the electronic excitation energy transfer (EET) processes. The singlet or triplet state of oxygen can also be quenched by the ground state triplet oxygen $\mathrm{O}_{2}\left({ }^{3} \sum_{\mathrm{g}}^{-}\right)$, forming the superoxide anion, $\left(\mathrm{O}_{2}{ }^{-}\right)$through the photo-induced electron transfer (PET) process ${ }^{11-13}$. The sequence shown in equations 1-4, illustrates the processes involved in the production of the above oxidants.

$$
\begin{aligned}
& \mathrm{PS} \stackrel{\mathrm{h} v}{\longrightarrow}{ }^{1} \mathrm{PS}^{*} \stackrel{\mathrm{ISC}}{\longrightarrow}{ }^{3} \mathrm{PS}^{*} \\
& { }^{3} \mathrm{PS}^{*}+\mathrm{O}_{2}\left({ }^{3} \sum_{g}^{-}\right) \stackrel{\mathrm{EET}}{\longrightarrow} \mathrm{PS}+\mathrm{O}_{2}\left({ }^{1} \Delta_{g}\right) \\
& { }^{3} \mathrm{PS}^{*}+\mathrm{O}_{2}\left({ }^{3} \sum_{g}^{-}\right) \stackrel{\mathrm{EET}}{\longrightarrow} \mathrm{PS}+\mathrm{O}_{2}\left({ }^{1} \sum_{g}^{+}\right) \\
& { }^{3} \mathrm{PS}^{*}+\mathrm{O}_{2}\left(\sum_{g}^{-}\right) \stackrel{\mathrm{PET}}{\longrightarrow} \mathrm{PS}^{+}+\mathrm{O}_{2}^{--}
\end{aligned}
$$

In this work, hematoxylin (HT), an aromatic compound containing phenolic OH groups (Figure 1a)was

* Corresponding author 
selected as a model compound for an organic pollutant in the aquatic environment. Hematoxylin is electroactive in the potential range accessible in the aqueous medium and hence it could be detected by cyclic voltammetry. Furthermore, HT is coloured and therefore its disappearance can be conveniently followed spectrophotometrically. Aqueous solutions of HT were irradiated with visible light, in the presence of oxygen dissolved in the solution, and the photosensitizer, protoporphyrin dimethylester (PPDME) (Figure 1b). The reactive species responsible for the photodegradation of HT have been identified. The photodegradation of HT was investigated using cyclic voltammetry and visible absorption spectroscopy.

\section{METHODS AND MATERIALS}

Protoporphyrin dimetylester (Aldrich), HT (Edward Gurr Ltd. London), sodium azide (Fluka, Switzerland) and 1,4-benzoquinone (BDH, UK) were used without further purification. All other chemicals and solvents used were commercial products of high purity. All aqueous solutions were prepared using deionized water (conductivity $<18 \mu \mathrm{S} \mathrm{cm}^{-1}$ ).

Photo-irradiation experiments were carried out using a home-made apparatus consisting of a $100 \mathrm{~W}$ tungsten filament lamp of radiant flux of $60 \mathrm{Wm}^{-2}$. The radiation emitted by this source was focused to the wall of a 50 $\mathrm{cm}^{3}$ Pyrex beaker containing the reaction mixture. The radiation from the lamp was filtered through a circulatory water jacket placed between the source and the reaction vessel, which avoided the warming of the solution. During irradiation, the samples were continuously bubbled with either oxygen or nitrogen as desired.

UV-Visible spectra were recorded on a Shimadzu1601 spectrophotometer. All cyclic voltammetric (cv) experiments were performed in a one-compartment cell comprising of a glassy carbon disk $\left(0.385 \mathrm{~cm}^{2}\right)$ working electrode, an $\mathrm{Ag}(\mathrm{s}) / \mathrm{AgCl}(\mathrm{s}) /$ sat. $\mathrm{KCl}$ (aq) reference electrode, and a platinum $(\mathrm{Pt})$ gauze counter electrode. Voltage scanning was done with a potentiostat (Oxford



Figure 1: The chemical structure of hematoxylin (a) and protoporphyrin dimethylester (b).
Instruments) and the signals were recorded on a BAS X-Y recorder (USA). An Orion model 420A pH/mV meter was used where $\mathrm{pH}$ adjustments were required.

In cv experiments, the working electrode was polished, prior to use, with $0.05 \mu \mathrm{m}$ alumina slurry and then cleaned with distilled water. The electrode was pre-treated by a continuous cyclic potential sweep from $-0.2 \mathrm{~V}$ to $+0.8 \mathrm{~V}$ (vs. $\mathrm{Ag} / \mathrm{AgCl}$ electrode) at $50 \mathrm{mV} \mathrm{s}^{-1}$, in acetate buffer solution ( $\mathrm{pH} 4.0)$ until a constant background current was observed. The solutions were deoxygenated, when necessary, by purging with nitrogen gas for $30 \mathrm{~min}$ prior to electrochemical measurements, and a blanket of nitrogen was maintained above the solution during measurements. Cyclic voltammograms were recorded for $0.1 \mathrm{mM} \mathrm{HT}, 0.1 \mathrm{mM}$ catechol on bare glassy carbon and PPDME-coated glassy carbon electrodes, in $\mathrm{pH} 4.0$ acetate buffer. Photo-oxidation studies of $1 \mathrm{mM}$ HT were carried out under steady-state irradiation, and samples were analyzed at regular time intervals by spectrophotometry and cyclic voltammetry for oxygen saturated, air-saturated and nitrogen saturated solutions, in the presence and absence of the photosensitizer. The experiments were repeated with and without 0.01 $\mathrm{M}$ sodium azide and also with and without $0.1 \mathrm{mM}$ benzoquinone.

\section{RESULTS AND DISCUSSION}

\section{Electrochemical behaviour of $\mathrm{HT}$ and PPDME at pH 4.0}

The voltammetric response of HT in acetate buffer at $\mathrm{pH}$ 4.0 on polished glassy carbon electrode shows two redox couples in the potential window of $-0.1 \mathrm{~V}$ to $+0.7 \mathrm{~V}$ at a

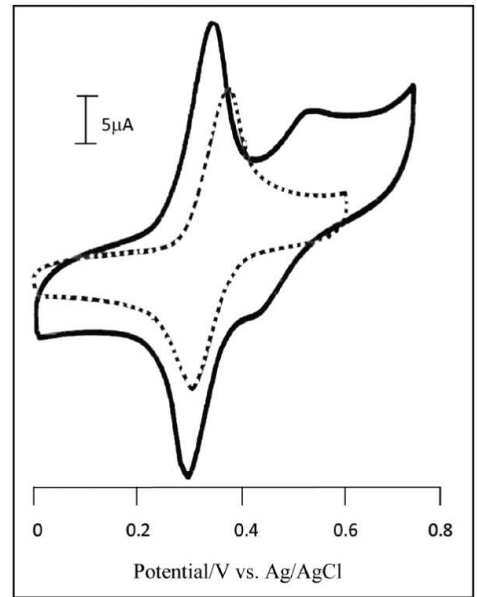

Figure 2: Cyclic voltammograms obtained on a glassy carbon electrode in $0.1 \mathrm{M}$ acetate buffer ( $\mathrm{pH} 4.0$ ) containing (a) $0.1 \mathrm{mM}$ HT (solid line), (b) $0.2 \mathrm{mM}$ catechol (dashed line), at a scan rate of $50 \mathrm{mV} \mathrm{s}^{-1}$. 
scan rate of $50 \mathrm{mV} \mathrm{s}^{-1}$ (Figure 2a). One of them is a well defined redox couple, centred at the peak potentials of $0.33 \mathrm{~V}$ and $0.29 \mathrm{~V}$. These peak potentials are comparable with those of $0.34 \mathrm{~V}$ and $0.30 \mathrm{~V}$, obtained for catechol under identical conditions (Figure $2 b$ ). Therefore, they could be attributed to the electrochemical oxidation/ reduction of ortho-phenolic groups far removed from the heterocyclic ring of HT. The second peak was a weak couple giving peak potentials at $0.5 \mathrm{~V}$ and $0.45 \mathrm{~V}$. This may correspond to the redox behaviour of the ortho-phenolic groups that are in close proximity to the heterocyclic ring.

Figure 3 shows the dependence of peak currents in CVs, obtained at different concentrations of HT. The oxidation current densities increased linearly with the HT concentration. CVs were recorded five times at a given concentration in order to confirm the reproducibility of the voltammetric responses. The plot of peak current density against concentration is a straight line, and hence it can be used as a calibration plot for the quantitative analysis of HT in the irradiated samples. Each point in the plot is an average of such five individual current density measurements. The electrochemical response of PPDME-coated glassy carbon electrode in acetate buffer $(\mathrm{pH}=4.0)$ consisted of an irreversible anodic peak current at $0.67 \mathrm{~V}$ vs $\mathrm{Ag} / \mathrm{AgCl}$ reference electrode.

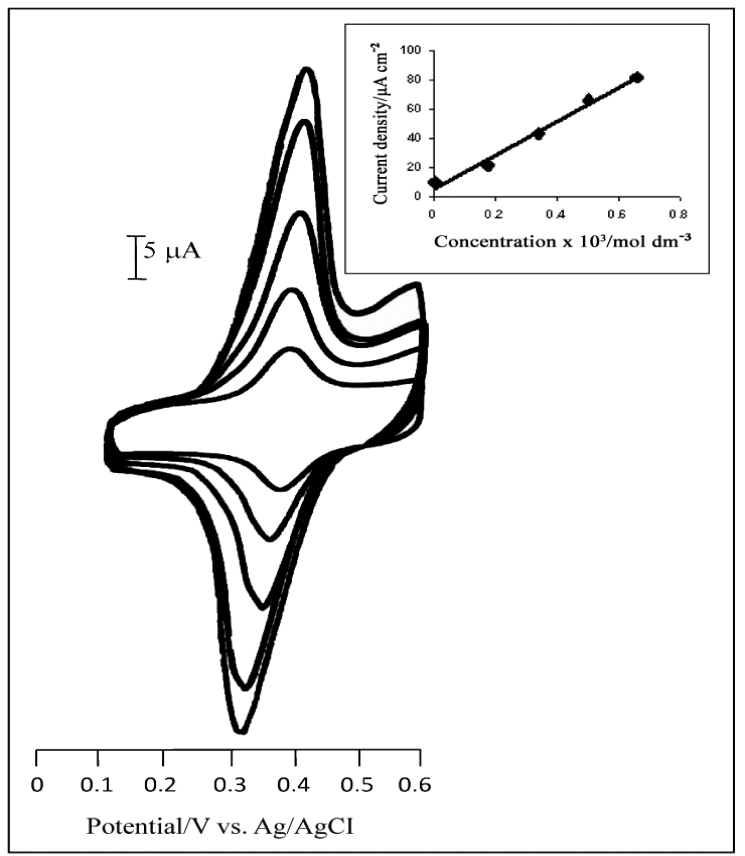

Figure 3: Cyclic voltammograms of HT, when its concentration is varied from $0.005 \mathrm{mM}$ to $0.750 \mathrm{mM}$ in the acetate buffer ( $\mathrm{pH} 4.0)$ on a glassy carbon electrode at a scan rate of $50 \mathrm{mV} \mathrm{s}^{-1}$. The inset shows the plot of current density vs. concentration of HT.

Journal of the National Science Foundation of Sri Lanka 35 (4)

\section{Photo-oxidation of HT}

In oxygenated solutions, complete disappearance of the initial magenta colour of HT within a two-hour period of irradiation in the presence of PPDME was observed. Thereafter the development of a yellow colour was visualized. Consequently, the absorbance at $560 \mathrm{~nm}$ was decreased and an absorption peak at $380 \mathrm{~nm}$ was progressively increased (Figure 4.1). Within two hours of irradiation, the magenta colour of HT (1) changes into the yellow colour of quinone (II) which corresponds

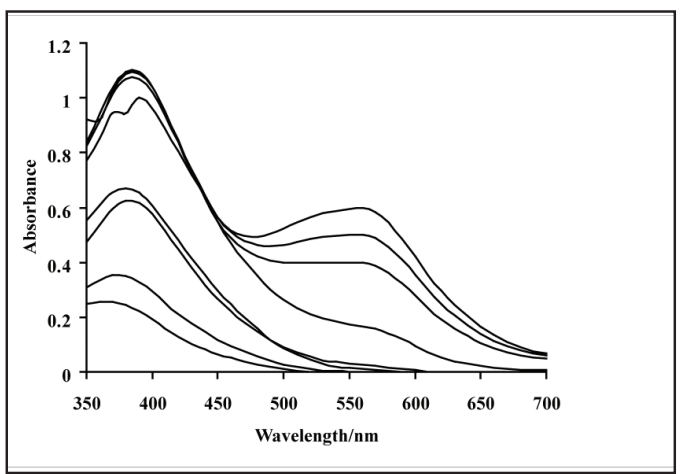

Figure 4.1: Absorption spectra of HT $(1.0 \mathrm{mM})$ as a function of visible light irradiation over 15 hours.

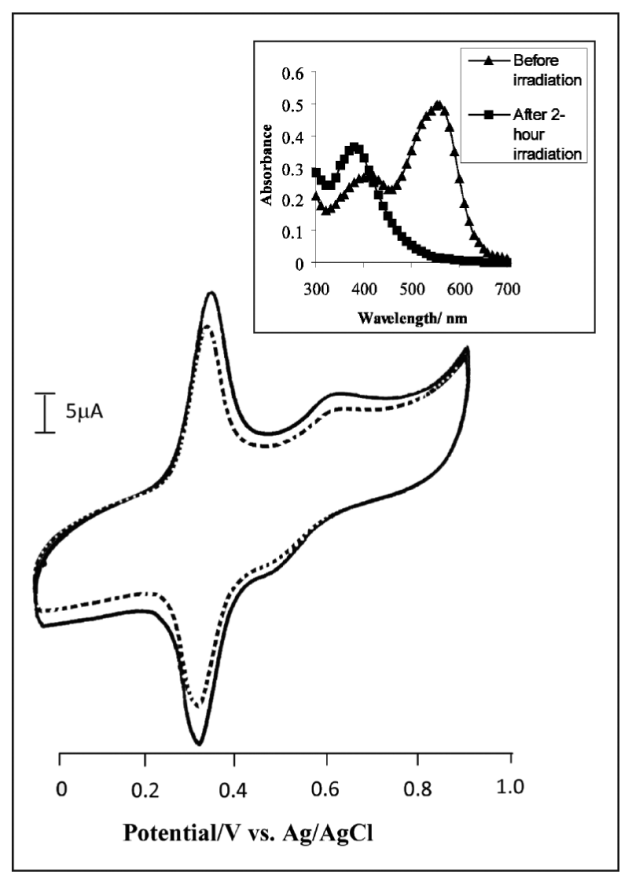

Figure 4.2: Cyclic voltammograms of HT in acetate buffer ( $\mathrm{pH}$ 4.0) before the irradiation (solid line) and after two hours of irradiation (dashed line), at a scan rate of $50 \mathrm{mV} \mathrm{s}^{-1}$. The inset shows the corresponding absorption spectra as a function of time. 


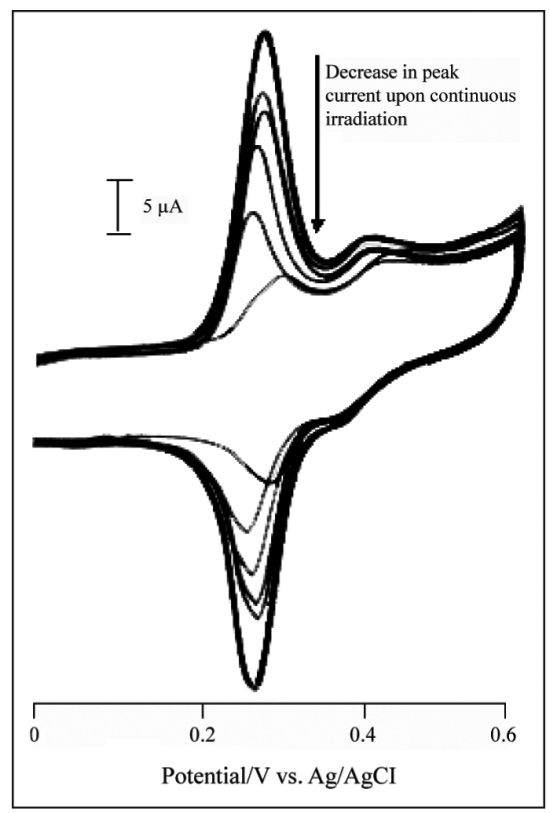

Figure 4.3: Cyclic voltammograms recorded during a 25 hour period of photo-oxidation of HT $(0.6 \mathrm{mM})$ in $0.1 \mathrm{M}$ acetate buffer ( $\mathrm{pH} 4.0$ ), at a scan rate of $50 \mathrm{mV} \mathrm{s}^{-1}$.

to the oxidation of HT as seen by the disappearance of visible absorption at $560 \mathrm{~nm}$ and the appearance of a new absorption at $380 \mathrm{~nm}$. The CV studies (Figure 4.2) indicate that, during this period, $17 \%$ of the quinone (II) had undergone further oxidation.

Upon further irradiation, the peak currents of the CVs decreased nearly to the background level (Figure 4.3), and the yellow colour of the solution was also discharged. In air saturated solutions, the same results were observed with a lower photo-oxidation rate. In the absence of light or nitrogen saturated illuminated solutions, photobleaching was not observed. It was also found that the photo-oxidation of HT, in the absence of PPDME, could take place but at a very slow rate upon prolonged irradiation (Figure 5).

To ascertain the possible involvement of reactive oxygen species in the oxidation process of HT, a well known singlet oxygen quencher, sodium azide, and a superoxide anion quencher, 1,4-benzoquinone (BQ), were employed. Figure 6 presents the change in absorbance of HT in pH 4.0 buffer, at $560 \mathrm{~nm}$ upon visible light irradiation, when $0.2 \mathrm{mM}$ 1,4-benzoquinone (BQ) or $0.1 \mathrm{M}$ sodium azide is present. In the presence of $\mathrm{BQ}$, complete retardation of the bleaching of the initial colour of HT was observed. A considerable change in colour was noticed for the azide ion containing solution while total photobleaching was observed when a quencher



Figure 5: Change in absorbance, measured at $560 \mathrm{~nm}$, for the disappearance of magenta colour of HT, upon irradiation under different conditions.<smiles>O=C1C=CC(=O)C=C1</smiles>

was absent in the oxygenated solutions. Benzoquinone is a well known superoxide radical quencher, by which a rapid single electron transfer takes place with $\mathrm{O}_{2}{ }^{-16}$ generating ground state oxygen, and benzoquinone radical anion (Reaction 5).

In the presence of azide ions as the singlet oxygen quencher ${ }^{17}$, photobleaching of HT takes place. However, when BQ is present, the rate of photobleaching is significantly decreased. These observations suggest that the reactive oxygen species responsible for photobleaching of HT is superoxide and not singlet oxygen. The benzoquinone radical formation would inhibit the participation of $\mathrm{O}_{2}^{--}$in the photo-oxidation of HT.

From a thermodynamic point of view, an electron transfer from the ground state of PPDME to $\mathrm{O}_{2}\left({ }^{3} \sum_{g}^{-}\right)$is not energetically favoured due to the high redox potential of the PPDME/PPDME ${ }^{+}$couple, as compared to the potential of $-0.16 \mathrm{~V}$ for the one electron reduction of ground state oxygen. However the oxidation potential recorded by $\mathrm{CV}$ for PPDME is $+0.86 \mathrm{~V}$, and the estimated oxidation potential for the excited couple $\left(\mathrm{PPDME}^{*} / \mathrm{PPDME}^{+}\right.$) is $-1.6 \mathrm{~V}$ (vs. NHE). These data clearly show the possibility of an electron transfer from the excited PPDME molecule to the ground state 


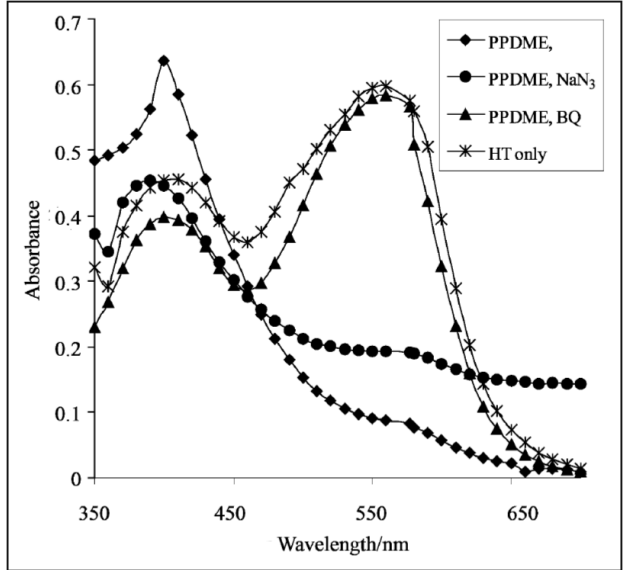

Figure 6: Spectral profiles showing the scavenging effect of BQ and $\mathrm{NaN}_{3}$ on photobleaching of air-saturated solution of HT under 2 hour irradiation.

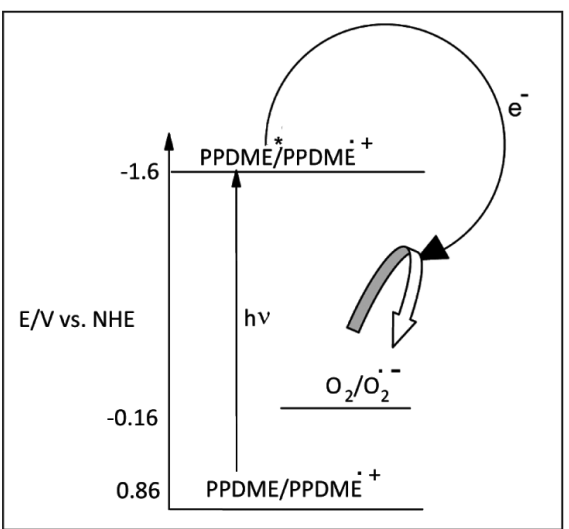

Figure 7: Energy-level diagram showing the formation of superoxide anion through the quenching of excited PPDME by ground state oxygen.

oxygen in order to generate a superoxide radical ion (Figure 7). The excited state redox potential of PPDME was estimated by the equation:

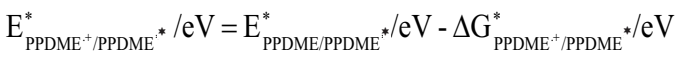

where $\Delta \mathrm{G}_{\text {PPDME/PPDME }}^{*}$ is the standard free energy difference, in $\mathrm{eV}$, between the excited state and the ground state of PPDME. Since the absorption threshold of PPDME occurs at $630 \mathrm{~nm}, \Delta \mathrm{G}_{\text {PPDME/PPDME }}^{*}{ }^{*}=2.5 \mathrm{eV}$.

The equilibrium constant of the reaction (7) at $25^{\circ} \mathrm{C}$ is reported to be $7.7 \times 10^{4} \mathrm{dm}^{3} \mathrm{~mol}^{-1}\left(\mathrm{pK}_{\mathrm{a}}=4.9\right)^{16}$. Therefore, superoxide ions in the acidic medium are almost completely converted to $\mathrm{HO}_{2}{ }^{\cdot}$ (Reaction 7) which disproportionates to form $\mathrm{H}_{2} \mathrm{O}_{2}$ (Reaction 8). Therefore, at $\mathrm{pH} 4.0, \mathrm{H}_{2} \mathrm{O}_{2}$ could also be an oxidant for the photo-oxidation of HT.
$\mathrm{O}_{2}^{-}+\mathrm{H}^{+} \rightleftharpoons \mathrm{HO}_{2}$

$2 \mathrm{HO}_{2} \rightleftharpoons \mathrm{O}_{2}+\mathrm{H}_{2} \mathrm{O}_{2}$

Since the superoxide ion is a good proton abstractor ${ }^{17}$, it can remove protons from the acidic phenolic groups of HT generating an oxidized product of HT (Reaction 9).

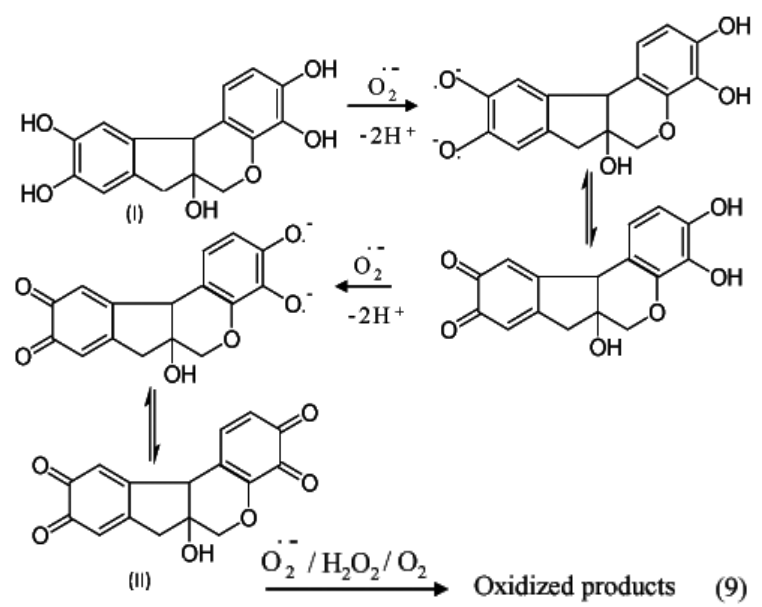

The yellow colour of the solution also decreases as a result of continued irradiation in the presence of the sensitizer and oxygen. This accompanies a subsequent decrease in absorbance at $380 \mathrm{~nm}$ down to the base line. The peaks in the CVs also gradually decrease, indicating that the redox sites are also degraded by the superoxide radicals formed. After 24 hours of illumination, the solution becomes almost colourless indicating that the complete disappearance of HT by superoxide radicals.

\section{CONCLUSION}

The degradation of hematoxylin in aqueous oxygenated solutions at $\mathrm{pH} 4.0$ has been achieved by means of photosensitization using PPDME as the sensitizer. It was found that the superoxide anion is the major oxidant in the destruction process of hematoxylin. However, singlet oxygen alone has less of an effect.

\section{Acknowledgement}

Authors wish to acknowledge the financial assistance from the National Science Foundation of Sri Lanka.

\section{References}

1. Schnoor J.L. (1991). Fate of Pesticides and Chemicals in the Environment. John Wiley, New York.

2 Bandara J., Morrison C., Kiwi J., Pulgarin C. \& Peringer P. (1996). Degradation/ decolouration of concentrated 
solutions of Orange II. Kinetics and quantum yield for sunlight induced reactions via Fenton type reagents. Journal of Photochemistry and Photobiology A: Chemistry 99: 57-63.

3. Chen R. \& Pignatello J. (1997). Role of quinine intermediates as electron shuttles in fenton and photoasisted fenton oxidations of aromatic compounds. Journal of the Environmental Science and Technology 31: 2399.

4. Legrini O., Oliveros E. \& Braun A.M. (1993). Photochemical processes for water treatment. Chemical Review 93: 671-674.

5. Vincze L. \& Kemp T.J. (1995). Light flux and light flux density dependence of the photomineralization rate of 2,4-dichlorophenol and chloroacetic acid in the presence of $\mathrm{TiO}_{2}$. Journal of Photochemistry and Photobiology A: Chemistry 87.

6. Sivakumar T. \& Shanthi K. (2000). Kinetic studies on the photo decolourisation of textile dyes (reactive) using $\mathrm{ZnO}$ catalyst. Indian Journal of Chemical Technology 7: 121-126.

7. Hoffmann M.R., Martin S.T., Chol W. \& Bahnemann D.W. (1995). Environmental application of semiconductor Photocatalysis. Chemical Review 95: 69-96.

8. Gerdes R., Wohrle D., Spiller W., Schneider G., Schnurpfeil G. \& Schulz-Ekloff G.J. (1997). Photo-oxidation of phenol and monochlorophenols in oxygen-saturated aqueous solutions by different photosensitizers. Journal of Photochemistry and Photobiology A: Chemistry 111: 65-74.

9. Nowakowska M. \& Szczubialka K. (1995). Photosensitized dechlorination of polychlorinated phenols Journal of Photochemistry and Photobiology A: Chemistry 91:81-85.

10. Randby B. \& Rabek J.F. (1975). Photo-degradation, Photo-oxidation and Photo-stabilization of Polymers. P. 254, John Wiley, New York.

11. Rodgers M.A.G. \& Snowden P.T. (1982). Lifetime of $\mathrm{O}_{2}\left({ }^{1} \Delta_{\mathrm{g}}\right)$ in liquid water as determined by time resolved infrared luminescence measurements. Journal of the American Chemical Society 104: 5541-5543.

12. Gandin E., Lion Y. \& Van de Vorst A. (1983). Quantum yield of singlet oxygen production by xanthene derivatives. Journal of Photochemistry and Photobiology A: Chemistry 37: 271.

13. Wang B. \& Ogilby P.R. (1995). Fraction of excited-state oxygen formed as $\mathrm{b}^{1} \sum_{\mathrm{g}}+$ in solution-phase photosensitized reactions. Journal of Photochemistry and Photobiology A: Chemistry 90: 85-89.

14. Peters G. \& Rodgers M. A. J. (1981). Single - electron transfer from NADH analogues to singlet oxygen. Biochimica Biophysica Acta 43: 637.

15. Cline Li M.Y.C.S., Koker E.B., Carmichael H.H., Chignell C.F. \& Bilski P. (1992). Quenching of singlet molecular oxygen $\left({ }^{1} \mathrm{O}_{2}\right)$ by azide anion in solvent mixtures. Journal of Photochemistry and Photobiology A: Chemistry 74: 760-764.

16. Bruceking R. (1994). Encyclopedia of Inorganic Chemistry. p.2951. John Wiley, London.

17. Moro-Oka Y. \& Foote C.S. (1976). Chemistry of superoxide ion. Oxidation of 3,5-Di-tert-butyleatechol with $\mathrm{KO}_{2}$. Journal of the American Chemical Society 98: 1510. 